\title{
Ramond-Ramond couplings of D-branes
}

\author{
Koji Hashimoto, ${ }^{a, b}$ Sotaro Sugishita ${ }^{c}$ and Seiji Terashima ${ }^{d}$ \\ ${ }^{a}$ Department of Physics, Osaka University, \\ Toyonaka, Osaka 560-0043, Japan \\ ${ }^{b}$ Mathematical Physics Lab., RIKEN Nishina Center, \\ Saitama 351-0198, Japan \\ ${ }^{c}$ Department of Physics, Kyoto University, \\ Kyoto 606-8502, Japan \\ ${ }^{d}$ Yukawa Institute for Theoretical Physics, Kyoto University, \\ Kyoto 606-8502, Japan \\ E-mail: koji@phys.sci.osaka-u.ac.jp, \\ sotaro@gauge.scphys.kyoto-u.ac.jp, terasima@yukawa.kyoto-u.ac.jp
}

ABSTRACT: Applying supersymmetric localization for superstring worldsheet theory with $\mathcal{N}=(1,1)$ supersymmetries on a cylinder and with arbitrary boundary interactions, we find the most general formula for the Ramond-Ramond (RR) coupling of D-branes. We allow all massive excitations of open superstrings, and find that only a finite number of them can contribute to the formula. The formula is written by Quillen's superconnection which includes higher form gauge fields, and the resultant general Chern-Simons terms are consistent with RR charge quantization. Applying the formula to boundary string field theory of a BPS D9-brane or a D9-antiD9 brane system, we find that any D9-brane creation via massive mode condensation is impossible.

Keywords: D-branes, Superstrings and Heterotic Strings, Gauge Symmetry, String Field Theory

ArXiv ePrint: 1501.00995 


\section{Contents}

1 Introduction: most general Ramond-Ramond charge formula 1

2 Localization in $(1,1)$-supersymmetric worldsheet theory 3

3 No creation of more D-branes $\quad 6$

3.1 The question 6

$\begin{array}{lll}3.2 & \text { BSFT for a BPS D9-brane } & 6\end{array}$

$\begin{array}{ll}3.3 & \text { BSFT for a D9-antiD9 pair }\end{array}$

4 General RR-coupling formula $\quad 11$

4.1 General RR-coupling formula for a BPS D9-brane 11

$\begin{array}{lll}4.2 & \text { General RR-coupling formula for multiple D9-branes } & 12\end{array}$

$\begin{array}{lll}5 & \text { Summary and discussion } & 17\end{array}$

\section{Introduction: most general Ramond-Ramond charge formula}

Identifying the Ramond-Ramond (RR) coupling of D-branes in superstring theory was the ignition of the second revolution by J. Polchinski [1]. Since then, tremendous amount of research has been carried out based on the D-brane RR-charge formula: the coupling between the RR field in the bulk and the massless open string degrees of freedom on multiple $\mathrm{D} p$-branes is $[2,3]$

$$
S_{\mathrm{RR}}=T_{q} \sum_{p} \int C_{\mathrm{RR}}^{(p+1)} \wedge \operatorname{Tr} e^{2 \pi \alpha^{\prime} F}
$$

where $F=d A-i A^{2}$ is a two-form field strength of a massless Yang-Mills gauge field on the D-branes. $T_{q}$ is the tension of a $\mathrm{D} q$-brane and $C_{\mathrm{RR}}^{(p+1)}$ is the $\mathrm{RR}(p+1)$-form field, where $p$ is an odd (even) integer for type IIB (IIA) superstring theory. Since the RR coupling formula (1.1) generates Chern characters for the Yang-Mills fields, the formula is consistent with RR charge quantization.

The full structure of the RR charge formula has not been addressed, because of complexity of the string excitations. In fact, the formula (1.1) should be able to be generalized to include all the open string excitations, even if we restrict our attention to the lowest tree-level in perturbation of string theory (that is a disk amplitude). In this paper, we provide a completely general formula for the RR coupling of D-branes, with arbitrary number of all massive open string excitations.

A part of the generalized RR coupling was widely known $[4,5]$ in the context of tachyon condensation in string theory. Starting with a pair of a D-brane and an anti-D-brane (or a non-BPS D-brane), one can include tachyon modes in addition to the massless modes. The 
RR coupling formula including the tachyon modes was given in the context of boundary string field theory (BSFT) [6-9]. For superstring theory, the action of the off-shell open string field theory is given by just a disk partition function with arbitrary boundary vertex insertions allowed [10-13]. The resultant RR coupling formula is written with the YangMills fields and the tachyon fields, and it was used to show Sen's conjecture [14-18] on D-brane annihilation via tachyon condensation. ${ }^{1}$

Any non-perturbative formulation of superstring theory needs to be capable of describing a D-brane creation, as well as the D-brane annihilation. As one can naively guess, to describe a D-brane creation, it is plausible to have a condensation of open string massive modes, rather than the tachyon condensation. The mass squared of the open string modes signals the direction of the energy uplift / decrease. So, to investigate whether open superstring theory can describe multiple D-brane creation in its off-shell configuration space or not, we need to generalize the previously known RR charge formula (1.1) and an analogous one including the tachyons, to the one with all massive open string modes. ${ }^{2}$

The necessary technique to calculate the most general RR charge formula is indeed ready, thanks to the recent developments on supersymmetric localization technique. (See, for example, [22]-[43]. For manifolds with boundaries, see [44]-[47].) As we mentioned, the RR charge formula at off-shell superstring theory is nothing but a worldsheet partition function. We apply the supersymmetric localization to the supersymmetric worldsheet theory with arbitrary number of arbitrary massive open string interactions at the worldsheet boundary.

The type II superstring worldsheet theory has $\mathcal{N}=(1,1)$ supersymmetries. We are interested in a flat target space-time for the moment, as we need to know what is the RR field configuration; at the flat spacetime, the RR field can be constant, which suits our purpose. Therefore the worldsheet theory has just chiral multiplets in $1+1$ dimensions. Nevertheless, we need to allow arbitrary interactions at the worldsheet boundary, which breaks only a half of the $\mathcal{N}=(1,1)$ supersymmetries on the worldsheet. We develop the localization technique for the theory and obtain an exact partition function with the arbitrary boundary interactions. ${ }^{3}$ Resultantly, we obtain the most general $R R$ charge formula in flat spacetime: ${ }^{4}$

$$
S_{\mathrm{RR}}=T_{q} \sum_{p} \int C_{\mathrm{RR}}^{(p+1)} \wedge \operatorname{Str} e^{2 \pi \alpha^{\prime} \mathcal{F}}
$$

This most general RR charge formula is written beautifully in terms of Quillen's superconnection $\mathcal{A}[50,51]$, with the field strength $\mathcal{F} \equiv d \mathcal{A}-i \mathcal{A}^{2}$. The superconnection includes

\footnotetext{
${ }^{1}$ In the BSFT, the descent relations in the Sen's conjecture were also proved [19].

${ }^{2} \mathrm{~A}$ treatment of all massive open string modes in BSFT was studied in detail in [20]. For a bosonic BSFT, condensation of massive modes was analyzed in [21].

${ }^{3}$ Note that these generically break the space-time SUSY.

${ }^{4}$ In curved spacetime, it is needed to include the curvature coupling term [48]. For BPS states in supersymmetric background, we can define the central charges from which also the quantized charges may be extracted. They were also computed using the localization technique $[41,45,46]$, although the massive modes were not included. The curvature coupling term in the central charge is represented by the Gamma class. (See, for example, [49].)
} 
higher form fields which are massive open superstring excitations. It also includes tachyon part for the case of the D9-antiD9 system which was conjectured in [52] and derived in $[4,5]$.

The open superstring massive modes show up in our general RR charge formula such that the quantization of the RR charge is ensured. This is natural but surprising, as only a limited number of open string excitation modes can enter the RR charge formula. In fact, only a finite number of all the massive modes can appear in the general formula. For the RR charge formula for a BPS D-brane, only five of them (including the massless modes) show up.

From the exact partition function of the string worldsheet as a boundary superstring field theory, we can provide a proof of no D9-brane creation, starting from a D9-brane, or a D9-antiD9 pair. So, even with massive field condensation, one never obtains a creation of the D9-brane in the context of BSFT.

The organization of this paper is as follows. In section 2, we provide a supersymmetric localization for the $\mathcal{N}=(1,1)$-supersymmetric worldsheet theory with chiral matter multiplets. Then in section 3, we study the generic boundary interaction of the worldsheet with the localization, and find that it is impossible to generate a D9-brane charge starting from a single BPS D9-brane or from a D9-antiD9 pair. In section 4, we provide the most general RR charge formula with arbitrary massive open string excitations, and describe its relevance to Quillen's superconnection.

\section{Localization in $(1,1)$-supersymmetric worldsheet theory}

The localization computation in Euclidean 2-dimension space has been considered for $\mathcal{N}=$ $(2,2)$ SUSY theories. Here, we will consider an $\mathcal{N}=(1,1)$ SUSY theory on a 2-dimensional flat cylinder with a finite length. ${ }^{5}$ In this setting, we can include all fluctuations on Dbranes, which are not needed to be space-time supersymmetric. We will take the notations and conventions which are used in the Polchinski's text book and we will take $\alpha^{\prime}=2$ below. In the $2 \mathrm{~d}$ bulk which is assumed to be flat, we have a following bosonic superfield:

$$
\mathbf{X}^{\mu}=X^{\mu}+i \theta \psi^{\mu}+i \bar{\theta} \tilde{\psi}^{\mu}+\theta \bar{\theta} F^{\mu}
$$

where $\theta$ and $\bar{\theta}$ are independent 1-component fermionic coordinates. The SUSY operators are $Q=\partial_{\theta}-\theta \partial_{z}$ and $\bar{Q}=\partial_{\bar{\theta}}-\bar{\theta} \partial_{\bar{z}}$. The operators $D=\partial_{\theta}+\theta \partial_{z}$ and $\bar{D}=\partial_{\bar{\theta}}+\bar{\theta} \partial_{\bar{z}}$ commute with $Q$ and $\bar{Q}$. Then, the SUSY transformation of $\mathbf{X}^{\mu}$ is explicitly given by

$$
\begin{aligned}
\delta X^{\mu} & =i \epsilon \psi^{\mu}+i \bar{\epsilon} \tilde{\psi}^{\mu}, \\
\delta \psi^{\mu} & =-i \epsilon \partial_{z} X^{\mu}-i \bar{\epsilon} F^{\mu}, \\
\delta \tilde{\psi}^{\mu} & =-i \bar{\epsilon} \partial_{\bar{z}} X^{\mu}+i \epsilon F^{\mu}, \\
\delta F^{\mu} & =-i \epsilon \partial_{z} \tilde{\psi}^{\mu}+i \bar{\epsilon} \partial_{\bar{z}} \psi^{\mu},
\end{aligned}
$$

\footnotetext{
${ }^{5}$ Superstring partition functions at tree level are considered with a disk worldsheet. In this paper we treat cylinder instead of the disk as in [19], because the SUSY boundary conditions are simple. With appropriate boundary conditions, the disk and cylinder partition functions gives a same result for the onshell space-time fields which correspond to conformal boundary interactions. For the off-shell fields, we need a field redefinition.
} 
where $z=\tau+i \sigma$ and $\tau$ is the periodic coordinate of $S^{1}$ of the cylinder with the period $2 \pi$. For the bulk $2 \mathrm{~d}$ action, we can take general non-linear sigma model action of $\mathbf{X}^{\mu}$, but here for simplicity we consider a conventional superstring action

$$
S_{\text {worldsheet }}=\frac{1}{4 \pi} \int d z d \bar{z}\left[\partial_{\bar{z}} X^{\mu} \partial_{z} X^{\mu}+F^{\mu} F^{\mu}+\psi^{\mu} \partial_{\bar{z}} \psi^{\mu}+\tilde{\psi}^{\mu} \partial_{z} \tilde{\psi}^{\mu}\right] .
$$

At the boundary corresponding to the D-branes, a half of SUSY should be broken. We will take the unbroken SUSY such that $\epsilon=\bar{\epsilon}$. For this SUSY, we have

$$
\begin{aligned}
\delta X^{\mu} & =i \epsilon\left(\psi^{\mu}+\tilde{\psi}^{\mu}\right), \\
\delta \psi^{\mu} & =-i \epsilon\left(\partial_{z} X^{\mu}+F^{\mu}\right), \\
\delta \tilde{\psi}^{\mu} & =-i \epsilon\left(\partial_{\bar{z}} X^{\mu}-F^{\mu}\right), \\
\delta F^{\mu} & =-i \epsilon\left(\partial_{z} \tilde{\psi}^{\mu}-\partial_{\bar{z}} \psi^{\mu}\right) .
\end{aligned}
$$

A boundary condition which is consistent with this SUSY is

$$
\left.\partial_{\sigma} X^{\mu}\right|_{\mathrm{b}}=0,\left.\quad\left(\psi^{\mu}-\tilde{\psi}^{\mu}\right)\right|_{\mathrm{b}}=0,\left.\quad \partial_{\sigma}\left(\psi^{\mu}+\tilde{\psi}^{\mu}\right)\right|_{\mathrm{b}}=0,\left.\quad F^{\mu}\right|_{\mathrm{b}}=0,
$$

which is an off-shell extension of the Neumann boundary condition, i.e. boundary condition for the D9-branes (and anti-D9-branes). ${ }^{6}$ At the boundary with this Neumann boundary condition, the SUSY transformations for the non-zero fields are

$$
\begin{aligned}
\delta X^{\mu} & =i \epsilon \psi_{\mathrm{b}}^{\mu}, \\
\delta \psi_{\mathrm{b}}^{\mu} & =-i \epsilon \partial_{\tau} X^{\mu},
\end{aligned}
$$

where $\psi_{\mathrm{b}}^{\mu}=\psi^{\mu}+\tilde{\psi}^{\mu}$. Then, we can easily see that the boundary interactions constructed from the superfield including

$$
\mathbf{X}_{\mathrm{b}}^{\mu} \equiv X^{\mu}+i \theta_{\mathrm{b}} \psi_{\mathrm{b}}^{\mu}
$$

and $D_{b}=\partial_{\theta_{b}}+\theta_{b} \partial_{\tau}$ are invariant under the SUSY $Q_{b}=\partial_{\theta_{b}}-\theta_{b} \partial_{\tau}$. Here the supercoordinates are $\tau$ and $\theta_{\mathrm{b}}=(\theta+\bar{\theta}) / 2$. In this way, we can consider an arbitrary boundary interaction which preserves the half of the world sheet SUSY.

At the other boundary of the cylinder we will put the following boundary condition which is consistent with the SUSY:

$$
0=\left.\partial_{z} X_{n}^{\mu}\right|_{\mathrm{b}^{\prime}}=\left.\partial_{\bar{z}} X_{-n}^{\mu}\right|_{\mathrm{b}^{\prime}}=\left.\partial_{z}\left(\psi_{n}^{\mu}+\tilde{\psi}_{n}^{\mu}\right)\right|_{\mathrm{b}^{\prime}}=\left.\partial_{\bar{z}}\left(\psi_{-n}^{\mu}+\tilde{\psi}_{-n}^{\mu}\right)\right|_{\mathrm{b}^{\prime}}, \text { for } n>0
$$

where $X_{n}^{\mu} \equiv \int e^{-i n \tau} X^{\mu}$. With the on-shell condition for the free theory, i.e. $0=\partial_{z} \partial_{\bar{z}} X=$ $\partial_{z} \tilde{\psi}=\partial_{\bar{z}} \psi=F$, we can see that this boundary condition corresponds to the closed string

\footnotetext{
${ }^{6}$ With the on-shell condition for the free bulk theory, this set of the boundary conditions (2.5) is equivalent to the usual Neumann boundary condition of the superstring. Instead of (2.5), one can find a different set of boundary conditions which is consistent with SUSY: $\left.\partial_{\sigma} X^{\mu}\right|_{\mathrm{b}}=0,\left.\partial_{\sigma}\left(\psi^{\mu}+\tilde{\psi}^{\mu}\right)\right|_{\mathrm{b}}=0$. If we further impose $\left.\left(\psi_{0}^{\mu}-\tilde{\psi}_{0}^{\mu}\right)\right|_{\mathrm{b}}=0$, where $\psi_{0}=\int d \tau \psi$, then this set of the boundary conditions is consistent with SUSY and is equivalent to the usual boundary condition of the superstring at on-shell. We are allowed to use this instead of (2.5).
} 
vacua $|0\rangle_{\mathrm{RR}}$ in the $\mathrm{RR}$ sector because the raising operators correspond to the positive modes of $\partial_{z}(*)$ and the negative modes of $\partial_{\bar{z}}(*) .{ }^{7}$ We can also insert the fermion zero modes $\psi_{0}^{\mu}=\frac{1}{4 \pi} \int d \tau\left(\psi^{\mu}+\tilde{\psi}^{\mu}\right)$ which are SUSY invariants at the boundary. Then, the insertion of the following

$$
-i \sum_{p=\text { odd }} \frac{1}{(p+1) !} C_{\mu_{0} \cdots \mu_{p}}^{(p+1)}(2 i)^{-(p+1) / 2} \psi_{0}^{\mu_{0}} \cdots \psi_{0}^{\mu_{p}}
$$

corresponds to the RR state $|C\rangle_{\mathrm{RR}}$ where

$$
C=\sum_{p=\text { odd }} C^{(p+1)}, \quad C^{(p+1)} \equiv \frac{1}{(p+1) !} C_{\mu_{0} \cdots \mu_{p}}^{(p+1)} d x^{\mu_{0}} \cdots d x^{\mu_{p}}
$$

is the background constant $\mathrm{RR}(p+1)$-form [4].

Now our field theory on the cylinder is SUSY invariant, thus we can use the localization technique. We define

$$
\begin{aligned}
V & =\left(\delta \psi^{\mu}\right)^{\dagger} \psi^{\mu}+\left(\delta \tilde{\psi}^{\mu}\right)^{\dagger} \tilde{\psi}^{\mu} \\
& \equiv i\left(\partial_{\bar{z}} X^{\mu}+F^{\mu}\right) \psi^{\mu}+i\left(\partial_{z} X^{\mu}-F^{\mu}\right) \tilde{\psi}^{\mu},
\end{aligned}
$$

then $\delta \int d \tau d \sigma \delta V=\int d \tau d \sigma \partial_{\tau} V=0$ and

$$
\delta V=2 \epsilon\left(\partial_{\bar{z}} X^{\mu} \partial_{z} X^{\mu}+F^{\mu} F^{\mu}+\psi^{\mu} \partial_{\bar{z}} \psi^{\mu}+\tilde{\psi}^{\mu} \partial_{z} \tilde{\psi}^{\mu}+\frac{i}{2} \partial_{\sigma}\left(\psi^{\mu} \tilde{\psi}^{\mu}\right)\right),
$$

is the usual free massless action (where the last term vanish with the boundary conditions and the saddle point equations). By adding the regulator action

$$
S_{\mathrm{reg}}=\left.t \int d \tau d \sigma \delta V\right|_{\epsilon=1}
$$

and taking $t \rightarrow \infty$ limit, we find saddle point equations

$$
0=\partial_{z} X^{\mu}=\partial_{\bar{z}} X^{\mu}=F^{\mu},
$$

and

$$
0=\partial_{z} \psi=\partial_{\bar{z}} \psi^{\mu}
$$

The latter means that the non-zero modes of the fermions can be put to be zero, since a rescaling of the fermions in the regulator action (2.13) to have a canonical kinetic term has the equations of motion (2.15).

The 1-loop determinant around the locus is trivial because the zero modes do not couple to the non-zero modes in the regulator action. Therefore, the supersymmetric localization tells us that in the evaluation of the partition function and physical observables we can drop the non-zero modes and can consider only the zero modes.

\footnotetext{
${ }^{7}$ Instead this, we can also take $0=\left.\partial_{z} X_{n}^{\mu}\right|_{\mathrm{b}^{\prime}}=\left.\partial_{\bar{z}} X_{-n}^{\mu}\right|_{\mathrm{b}^{\prime}}=\left.\partial_{z} \psi_{n}^{\mu}\right|_{\mathrm{b}^{\prime}}=\left.\partial_{z} \tilde{\psi}_{n}^{\mu}\right|_{\mathrm{b}^{\prime}}=\left.\partial_{\bar{z}} \psi_{-n}^{\mu}\right|_{\mathrm{b}^{\prime}}=\left.\partial_{\bar{z}} \tilde{\psi}_{-n}^{\mu}\right|_{\mathrm{b}^{\prime}}=$ $\left.\left(\left(\partial_{z}-\partial_{\bar{z}}\right) \partial_{z} X+2 \partial_{z} F\right)_{n}\right|_{\mathrm{b}^{\prime}}=\left.\left(\left(\partial_{z}-\partial_{\bar{z}}\right) \partial_{\bar{z}} X+2 \partial_{z} F\right)_{-n}\right|_{\mathrm{b}^{\prime}} \quad($ for $n>0)$ which is consistent with SUSY and equivalent to the closed string vacua with the on-shell condition for the free theory. Note that the both of the boundary conditions are not consistent with the SUSY with $\epsilon \neq \bar{\epsilon}$. However, at the on-shell this corresponds to the closed string vacua, thus there is no real problem and this will be an artifact of extending to the off-shell SUSY.
} 


\section{No creation of more D-branes}

\subsection{The question}

Our interest here is whether one can climb up the potential hill in the BSFT, to obtain a solution of the BSFT representing multiple D-branes, starting from a single D-brane. To be concrete, we consider only D9-branes as a starting point for the BSFT action. These include a BPS D9-brane, and a pair of a D9-brane and an anti-D9-brane (brane-antibrane pair).

First, we need to summarize what we have seen for the localization. After the localization, the vacuum expectation value of any supersymmetric operator at the worldsheet boundary can be evaluated at the localization locus,

$$
\dot{X}^{\mu}(\tau)=0, \quad \dot{\psi}^{\mu}(\tau)=0 .
$$

The dot denotes a derivative with respective to the boundary coordinate $\tau$ of the worldsheet. $\mu$ runs from 0 to 9 labeling the target spacetime dimensions. In the following we omit the suffix "b" for $\mathbf{X}_{\mathrm{b}}, \theta_{\mathrm{b}}$ and $\psi_{\mathrm{b}}$ in (2.7).

The generic boundary interaction is written in terms of the superfield in 1 dimension (the boundary of the worldsheet),

$$
\mathbf{X}^{\mu} \equiv X^{\mu}(\tau)+i \theta \psi^{\mu}(\tau),
$$

and we allow arbitrary supersymmetric vertex operators, as BSFT is defined as a complete set of them.

Our aim is to show whether multiple D-branes can be created in the BSFT or not. We already know that the tachyon condensation can make a D-brane vanish, but the problem here is to see whether one can create one more unit of the D-brane charge by a condensation of some massive modes of string theory. See figure 1. We define "seeing the creation" by looking at the total D-brane charge. So, for example, creation of a brane-antibrane pair cannot be detected by our formalism.

Of course, it is easy to create a charge of a D-brane with different spatial world volume dimensions. For example, it is well-known that, on a BPS D-brane, turning on a constant magnetic field results in a different D-brane charge (of a D-brane with lower dimensions). So the question of our concern is: let us start with a D9-brane, and with a condensation of massive modes on the D9-brane, can we create a charge of multiple D9-branes?

There are two possible starting points. The first one is a single BPS D9-brane. The second one is a pair of BPS D9-brane and anti-D9-brane. The latter was used for the tachyon condensation giving the annihilation of the pair [5]. But in fact, for our purpose, nothing can prevent us from analyzing the first case. We check whether string theory can have a power to obtain multiple D-brane charges starting from the (naive) Hilbert space of a single D-brane.

\subsection{BSFT for a BPS D9-brane}

First, let us investigate the case of a single BPS D9-brane. The question is - can we create another D9-brane by making a condensation of massive modes on the BPS D9-brane? 

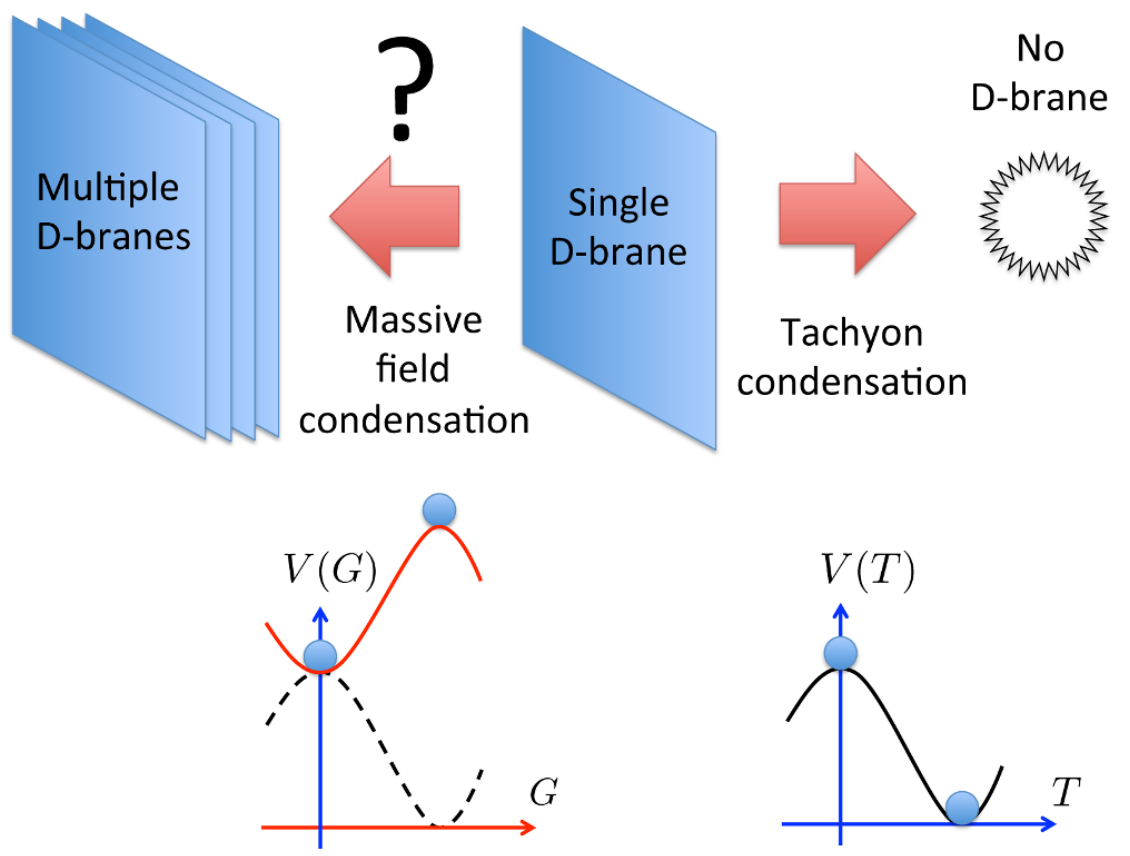

Figure 1. A schematic picture of the question of the D-brane creation. For the right part, a popular (and proven) D-brane annihilation by the tachyon condensation is shown. The left part is our question. To create multiple D-branes, more energy is necessary for the D-brane tensions, thus a condensation of massive modes $G$ which climbs up the potential hill would be naturally expected.

It would be instructive to review what would happen to the condensation of a massless mode. Later we will consider a massive mode, and study the generic case. The massless mode of an open string is the photon vertex operator,

$$
I_{\mathrm{B}}=\int d \tau d \theta\left(-i D_{\theta} \mathbf{X}^{\mu} A_{\mu}[\mathbf{X}]\right)
$$

If we make an integration of the boundary $\theta$ coordinate, we obtain

$$
I_{\mathrm{B}}=\int d \tau\left(-i \dot{X}^{\mu} A_{\mu}[X]+\frac{i}{2} \psi^{\mu} \psi^{\nu} F_{\mu \nu}[X]\right) .
$$

The BSFT action for the RR sector is nothing but the partition function of the worldsheet theory with some fermion zero modes corresponding to the background RR-forms, so basically we evaluate the expectation value of the operator $\exp \left[-I_{\mathrm{B}}\right]$. When evaluating the expectation value (VEV), we use the localization. The locus satisfies (3.1), so we substitute (3.1) into the boundary interaction, to have

$$
\left\langle e^{-I_{\mathrm{B}}}\right\rangle=\mathcal{N} \exp \left[-\frac{i}{2} \int d \tau \psi_{0}^{\mu} \psi_{0}^{\nu} F_{\mu \nu}\left[X_{0}\right]\right] .
$$

Note that the VEV \langle\rangle is defined for massive modes, and the zero modes $\psi_{0}$ and $X_{0}$ are not integrated yet. The overall constant $\mathcal{N}$ is can be evaluated by the localization explicitly but here we do not need it, as it will be a normalization of the RR charge. 
The expression (3.5) is a well-known formula for the RR charge. If the field strength $F_{\mu \nu}$ of the photon is constant, the expansion of the exponential form of (3.5) in terms of the field strength supplies multiple fermionic zero modes. Those zero modes are cancelled precisely by the RR vertex insertion, so we obtain lower dimensional D-brane charges. Note here that after the expansion of the exponential, the first term (the term without the fermion zero mode) is of course the unity. It means that the boundary state given by this boundary deformation has a unit RR charge for the D9-brane, in addition to the lower dimensional D-brane charges. So, we see here that the photon condensation can never give a RR charge of multiple D9-branes if we start from a D9-brane. The condensation is accompanied with fermionic zero modes $\psi_{0}$ in (3.5), thus creating only lower dimensional D-brane charges.

Next, let us visit an example of a concrete massive state. We consider a vertex for the first massive state on the BPS D9-brane (see for example [20]). The vertex is represented by a boundary action of the worldsheet,

$$
\begin{aligned}
I_{\mathrm{B}}=\int d \tau d \theta & \left(D_{\theta} \mathbf{X}^{\mu} D_{\theta} \mathbf{X}^{\nu} D_{\theta} \mathbf{X}^{\rho} V_{\mu \nu \rho}[\mathbf{X}]\right. \\
& \left.+D_{\theta}^{2} \mathbf{X}^{\mu} D_{\theta} \mathbf{X}^{\nu} W_{\mu \nu}[\mathbf{X}]+D_{\theta}^{3} \mathbf{X}^{\mu} S_{\mu}[\mathbf{X}]\right) .
\end{aligned}
$$

Here normally the arbitrary function $V, W$ and $S$ can be expanded by plane waves, and the indices $\mu, \nu$ and $\rho$ provides the polarization of the states. One can show that $S_{\mu}$ and the anti-symmetric part of $W_{\mu \nu}$ can be gauged away. So we need to consider only the symmetric part of $W_{\mu \nu}$ and the antisymmetric $V_{\mu \nu \rho}$.

Since the expression $I_{\mathrm{B}}$ is explicitly written only by the super field $\mathbf{X}$, the state is supersymmetric. After making the integration of the boundary fermion coordinate $\theta$, one obtains $I_{\mathrm{B}} \equiv I_{\mathrm{B}}[W]+I_{\mathrm{B}}[V]$ with $[20]$

$$
\begin{aligned}
I_{\mathrm{B}}[W] & =\int d \tau\left[\dot{X}^{\mu} \psi^{\nu} \psi^{\rho} \partial_{\rho} W_{\mu \nu}(X)+\left(\dot{X}^{\mu} \dot{X}^{\nu}-\dot{\psi}^{\mu} \psi^{\nu}\right) W_{\mu \nu}(X)\right] \\
I_{\mathrm{B}}[V] & =\int d \tau\left[\psi^{\mu} \psi^{\nu} \psi^{\rho} \psi^{\sigma} \partial_{\sigma} V_{\mu \nu \rho}(X)-3 \psi^{\mu} \psi^{\nu} \dot{X}^{\rho} V_{\mu \nu \rho}(X)\right] .
\end{aligned}
$$

Now, let us consider a condensation of the massive mode. This means that we have

$$
W_{(\mu \nu)}\left(X_{0}\right) \neq 0, \quad V_{[\mu \nu \rho]}\left(X_{0}\right) \neq 0 .
$$

Our interest is, for this condensation, whether we can have a RR-charge of the D9-brane or not. To evaluate the VEV of $e^{-I_{\mathrm{B}}}$, let us substitute the localization locus condition (3.1) into the boundary action. We obtain

$$
\left.I_{\mathrm{B}}\right|_{\text {locus }}=\int d \tau \psi_{0}^{\mu} \psi_{0}^{\nu} \psi_{0}^{\rho} \psi_{0}^{\sigma} \partial_{\sigma} V_{\mu \nu \rho}\left(X_{0}\right)
$$

It is obvious that this result has the same property as the case of the photon (3.5). All the condensation fields are accompanied by the fermion zero modes $\psi_{0}$, so they merely gives lower-dimensional D-brane charges. They never create the additional D9-brane charge. 
After examining some examples, we can come to a generic statement. On the BPS D9-brane, generic boundary action can be written as

$$
I_{\mathrm{B}}=\sum_{n_{1}, n_{2}, \ldots} \int d \tau d \theta f_{n_{1}, n_{2}, \cdots}[\mathbf{X}]\left(D_{\theta} \mathbf{X}\right)^{n_{1}}\left(\left(D_{\theta}\right)^{2} \mathbf{X}\right)^{n_{2}} \cdots\left(\left(D_{\theta}\right)^{k} \mathbf{X}\right)^{n_{k}} \cdots
$$

Using the component expression, we find

$$
D_{\theta} \mathbf{X}=i \psi+\theta \dot{X}, \quad D_{\theta}^{2} \mathbf{X}=\dot{X}+i \theta \dot{\psi}, \quad \cdots
$$

and

$$
f[\mathbf{X}]=f(X)+i \theta \psi \partial f[X]
$$

Then the boundary integral $d \theta$ is performed, under the condition of the localization locus (3.1), to give

$$
\left.I_{\mathrm{B}}\right|_{\text {locus }}=\sum_{n_{1}} \int d \tau \partial g_{n_{1}}\left[X_{0}\right]\left(\psi_{0}\right)^{n_{1}+1}
$$

where $g_{n_{1}} \equiv f_{n_{1}, n_{2}=n_{3}=n_{4}=\cdots=0}$. This is because only $D_{\theta} \mathbf{X}$ has a component without the time derivative, and the other $\left(D_{\theta}\right)^{k} \mathbf{X}$ with $k \geq 2$ vanishes due to (3.1). Since $n_{1}$ is a non-negative integer, the resultant (3.14) includes at least one fermionic zero mode $\psi_{0}$. Therefore, any condensation gives only lower-dimensional RR charges. This concludes a proof that, on a BPS D9-brane, condensation of any open string massive mode cannot change the D9-brane RR charge. Hence the BSFT cannot accommodate a D-brane creation starting from a single BPS D-brane.

\subsection{BSFT for a D9-antiD9 pair}

For the case of a pair of a D9-brane and an anti-D9-brane, we can generalize the proof found in the previous subsection. The BSFT action for a brane-antibrane was found in [5] and [4]. For the boundary interaction, the difference from the BPS D-brane studied above is just the inclusion of a fermionic boundary superfield

$$
\boldsymbol{\Gamma}=\eta(\tau)+\theta F(\tau)
$$

For the brane-antibrane pair, this field is complex, while for a non-BPS D-brane, this field is taken to be real [53] (see also [54, 55]).

Originally the boundary field $\eta$ was introduced by Witten [56] for the purpose of giving a Chan-Paton factor to represent the non-BPS D-brane before a worldsheet projection. So the inclusion of this $\boldsymbol{\Gamma}$ in the boundary interaction is basically just linear. The field $F(\tau)$ is an auxiliary field, since the kinetic term for this boundary superfield is

$$
S_{\boldsymbol{\Gamma}} \equiv-\int d \tau d \theta \overline{\boldsymbol{\Gamma}} D_{\theta} \boldsymbol{\Gamma}=\int d \tau[\bar{\eta} \dot{\eta}-\bar{F} F]
$$


Now, let us write a generic boundary interaction as before, with a possible linear component in $\boldsymbol{\Gamma}$, as $^{8}$

$$
\begin{aligned}
I_{\mathrm{B}}= & \sum_{n_{1}, n_{2}, \ldots} \int d \tau d \theta \boldsymbol{\Gamma} g_{n_{1}, n_{2}, \ldots}[\mathbf{X}]\left(D_{\theta} \mathbf{X}\right)^{n_{1}}\left(D_{\theta}^{2} \mathbf{X}\right)^{n_{2}} \cdots\left(D_{\theta}^{k} \mathbf{X}\right)^{n_{k}} \ldots+\text { c.c. } \\
& +\sum_{n_{1}, n_{2}, \ldots} \int d \tau d \theta \mathbf{\Gamma} \overline{\boldsymbol{\Gamma}} G_{n_{1}, n_{2}, \cdots}[\mathbf{X}]\left(D_{\theta} \mathbf{X}\right)^{n_{1}}\left(D_{\theta}^{2} \mathbf{X}\right)^{n_{2}} \cdots\left(D_{\theta}^{k} \mathbf{X}\right)^{n_{k}} \cdots
\end{aligned}
$$

The new part is $\boldsymbol{\Gamma}, \overline{\boldsymbol{\Gamma}}$ and $\boldsymbol{\Gamma} \overline{\boldsymbol{\Gamma}}$.

A popular example is a tachyon vertex operator. This corresponds to having $n_{1}=$ $n_{2}=\cdots=0$ for the first line in (3.17),

$$
I_{\mathrm{B}}[T]=\int d \tau d \theta \frac{1}{\sqrt{2 \pi}}(T[\mathbf{X}] \overline{\mathbf{\Gamma}}+\bar{T}[\mathbf{X}] \mathbf{\Gamma}) .
$$

So the general expression (3.17) includes the tachyon condensation as a particular case. The massless gauge fields on each D9-brane are also included.

Now, the localization locus condition (3.1) is applied to the vertex operator (3.17) as before, to obtain the following expression

$$
\begin{aligned}
\left.I_{\mathrm{B}}\right|_{\text {locus }}= & \sum_{n_{1}} \int d \tau\left(\partial g_{n_{1}}\left[X_{0}\right]\left(i \psi_{0}\right)^{n_{1}+1} \eta+g_{n_{1}}\left[X_{0}\right]\left(i \psi_{0}\right)^{n_{1}} F\right)+\text { c.c. } \\
& +\sum_{n_{1}} \int d \tau\left(\partial G_{n_{1}}\left[X_{0}\right]\left(i \psi_{0}\right)^{n_{1}+1} \eta \bar{\eta}+G_{n_{1}}\left[X_{0}\right]\left(i \psi_{0}\right)^{n_{1}}(F \bar{\eta}+\bar{F} \eta)\right) .
\end{aligned}
$$

Since we are interested in a D9-brane charge, only the choice $n_{1}=0$ is a candidate. (Other value of $n_{1}$ provides a creation of lower dimensional D-brane charge.) So dropping other terms gives ${ }^{9}$

$$
\left.I_{\mathrm{B}}\right|_{\text {locus }}=\sum_{n_{1}} \int d \tau\left(g_{n_{1}=0}\left[X_{0}\right] F+\text { c.c. }+G_{n_{1}=0}\left[X_{0}\right](F \bar{\eta}+\bar{F} \eta)\right) .
$$

This term would be a possible term which can create the D9-brane charge. However, the first term is nothing but the tachyon coupling (3.18). The second term can be generated by a field redefinition of the tachyon $T[X]$ (see the discussion in section 2 in [5]). So, this (3.20) is not a massive excitation of the open string - it is merely a tachyon coupling, which has been already shown to be unable to produce an additional D9-brane RR charge. ${ }^{10}$

This concludes a proof that a D9-brane charge cannot be generated by any condensation of massive excitation modes of open superstring in BSFT.

\footnotetext{
${ }^{8}$ On the brane-antibrane, there are vertex operators without the boundary super field $\boldsymbol{\Gamma}$. For that vertices, the discussion reduces to that of the previous subsection, so we do not treat those in the following.

${ }^{9}$ In the RR sector of the BSFT action, we need a supertrace, i.e. a factor $[\bar{\eta}, \eta]$ in the path integral measure for the $\eta$ and $\bar{\eta}$ integration [5].

${ }^{10}$ Since we assume the homogeneity for the D9-brane, the field $g_{n_{1}=0}\left[X_{0}\right]$ needs to be a constant.
} 


\section{General RR-coupling formula}

In the previous section, we have shown that the D-brane change cannot be created by any condensation of massive open strings. This applies only to the charge of the same kind of D-branes: in our previous cases those are D9-brane charges. On the other hand, lower-dimensional D-brane charges can be easily created. Here we shall derive a generic RRcharge formula including any massive mode condensation, which generalizes the known RR charge formula originally written only with condensation of the massless and the tachyonic modes on the D-brane(s).

\subsection{General RR-coupling formula for a BPS D9-brane}

For the massless mode condensation the popular expression for the RR coupling in the D-brane action is the Chern-Simons term (or often called "Wess-Zumino term"),

$$
S_{\mathrm{RR}}=T_{D 9} \sum_{p: \text { odd }} \int C^{(p+1)} \wedge e^{2 \pi \alpha^{\prime} F}
$$

where $C^{(p+1)}$ with an odd integer $p$ is the $\mathrm{RR}(p+1)$-form, and $F$ is the world volume gauge field strength which is 2 -form. The integrand in (4.1) is chosen in such a way that the total degree of the form is equal to the world volume dimension of the D-brane (which is 10 for the case of the BPS D9-brane). In the course of generalizing this formula, we just look back how this (4.1) was derived. The boundary action (3.5) is precisely the origin of the formula. A nonzero scattering amplitude after the path integral of the fermion zero mode $\psi_{0}$ requires an insertion of the $\mathrm{RR}$ vertex

$$
C_{\mu_{0} \mu_{2} \cdots \mu_{p}}^{(p+1)} \psi_{0}^{\mu_{0}} \cdots \psi_{0}^{\mu_{p}}
$$

so that the total number of the fermion zero mode is 10 (and completely antisymmetric under the exchange in variables $\mu_{i}$ ).

Now, let us generalize the formula to include a condensation of the open string massive modes. The result of the localization for the worldsheet boundary action is already given by (3.14). More specifically, the nonzero contribution to $I_{\mathrm{B}}$ after the localization comes only from a specific type of the open string excitation

$$
I_{\mathrm{B}}=-i \int d \tau d \theta g_{\mu_{1} \cdots \mu_{n}}^{(n)}[\mathbf{X}] D_{\theta} \mathbf{X}_{1}^{\mu} \cdots D_{\theta} \mathbf{X}^{\mu_{n}},
$$

where the indices $\mu_{1}, \cdots, \mu_{n}$ are mutually anti-symmetric. The integer $n$ should be an odd integer for a BPS D9-brane, due to the GSO projection. The $n=1$ case corresponds to the massless gauge field (3.3). The $n=3$ case is a part of the first excited massive mode (3.6). Following the same logic for the fermion zero mode integration, we arrive at a generic formula

$$
S_{\mathrm{RR}}=T_{D 9} \sum_{p: \text { odd }} \int C^{(p+1)} \wedge \exp \left[4 \pi \sum_{m=1}^{5} d g^{(2 m-1)}\right] .
$$


Here in the exponent, $d g^{(2 m-1)}$ is the $2 m$-form field strength of the open string massive mode $g^{(2 m-1)}(x)$,

$$
g^{(2 m-1)}=(-2 i)^{m-1} g_{\mu_{1} \cdots \mu_{2 m-1}}^{(2 m-1)}(x) d x^{\mu_{1}} \wedge \cdots \wedge d x^{\mu_{2 m-1}} .
$$

The numerical factors in the formula will be determined in a more general study in the next subsection.

The important point is that the RR coupling formula (4.4) is for all open string excitations. The open string fields appearing in the formula, $g^{(2 m-1)}$ with $m=1,2,3,4,5$ are the only fields which can contribute to the RR charge. In particular, we have found that the open string excitations which can contribute to the RR charge is restricted to mass level 4 .

\subsection{General RR-coupling formula for multiple D9-branes}

Next, we apply the same strategy for the D9-antiD9 pairs. We shall find that the most general RR coupling formula is written by the Quillen's superconnection including higher form fields.

Let us consider $N$ D9-D9bar branes with $N=2^{n-1}$. This system is realized by introducing the boundary auxiliary superfields $\Gamma^{i}$ where $i=1, \ldots, 2 n .{ }^{11}$ The action with general fluctuations at the boundary is

$$
-\int d \tau d \theta \frac{1}{4}\left(\boldsymbol{\Gamma}^{i} D_{\theta} \boldsymbol{\Gamma}^{i}\right)+I_{\mathrm{B}}(\boldsymbol{\Gamma}, \mathbf{X})
$$

where $I_{\mathrm{B}}$ is a general boundary action. The boundary action $I_{\mathrm{B}}$ does not include $D_{\theta} \boldsymbol{\Gamma}$ because $\boldsymbol{\Gamma}$ is an auxiliary field. Thus we can write $I_{\mathrm{B}}$ as

$$
I_{\mathrm{B}}=-i \int d \tau d \theta A_{n_{1}, n_{2}, \ldots ; m}(\mathbf{X})\left(D_{\theta} \mathbf{X}\right)^{n_{1}}\left(\left(D_{\theta}\right)^{2} \mathbf{X}\right)^{n_{2}} \cdots \times(\boldsymbol{\Gamma})^{m}
$$

where

$$
\begin{aligned}
A_{n_{1}, n_{2}, \ldots ; m}(\mathbf{X})\left(D_{\theta} \mathbf{X}\right)^{n_{1}} & \left(\left(D_{\theta}\right)^{2} \mathbf{X}\right)^{n_{2}} \cdots \times(\boldsymbol{\Gamma})^{m} \\
= & A_{\left(\mu_{1}^{1}, \mu_{2}^{1}, \cdots, \mu_{n_{1}}^{1}\right),\left(\mu_{1}^{2}, \mu_{2}^{2}, \cdots, \mu_{n_{2}}^{2}\right), \cdots ;\left(k_{1}, k_{2}, \cdots, k_{m}\right)}(\mathbf{X}) \\
& \times\left(D_{\theta} \mathbf{X}^{\mu_{1}^{1}} D_{\theta} \mathbf{X}^{\mu_{2}^{1}} \cdots D_{\theta} X^{\mu_{n_{1}}^{1}}\right) \\
& \times\left(\left(D_{\theta}\right)^{2} X^{\mu_{1}^{2}}\left(D_{\theta}\right)^{2} X^{\mu_{2}^{2}} \cdots\left(D_{\theta}\right)^{2} X^{\mu_{n_{2}}^{2}}\right) \cdots \\
& \times \boldsymbol{\Gamma}^{k_{1}} \boldsymbol{\Gamma}^{k_{2}} \cdots \boldsymbol{\Gamma}^{k_{m}}
\end{aligned}
$$

Here, $I_{\mathrm{B}}$ needs to be bosonic, thus $A=0$ if $\sum_{a=\text { odd }} n_{a}+m=2 \mathbf{Z}+1$. This condition is nothing but the GSO projection. We also impose the reality condition: $I_{\mathrm{B}}{ }^{*}=-I_{\mathrm{B}}$ in the Wick rotated world sheet action.

\footnotetext{
${ }^{11}$ A generalization to the non BPS D9-branes in type IIA superstring theory is easily achieved by taking $i=1, \ldots, 2 n+1$. Here we have moved to another notation for the gamma matrices for convenience. For $n=2$, they are related to the previous ones as $\boldsymbol{\Gamma}=(1 / 2)\left(\boldsymbol{\Gamma}^{1}+i \boldsymbol{\Gamma}^{2}\right)$ and $\overline{\boldsymbol{\Gamma}}=(1 / 2)\left(\boldsymbol{\Gamma}^{1}-i \boldsymbol{\Gamma}^{2}\right)$.
} 
We shall check infinitesimal gauge transformations to find a consistent non-Abelian nature of the boundary interaction written by $\boldsymbol{\Gamma}^{i}$. The gauge transformations are generated by adding the following general total divergence term to the boundary action,

$$
\begin{aligned}
0=-i \int d \tau d \theta D_{\theta} & \left(\lambda_{n_{1}, n_{2}, \cdots ; m}(\mathbf{X})\left(D_{\theta} \mathbf{X}\right)^{n_{1}}\left(D_{\theta} \mathbf{X}\right)^{n_{2}} \cdots \times(\boldsymbol{\Gamma})^{m}\right) \\
=-i \int d \tau d \theta( & \left(\frac{\partial}{\partial X^{\mu}} \lambda_{n_{1}, n_{2}, \cdots ; m}\right) D_{\theta} \mathbf{X}^{\mu}\left(D_{\theta} \mathbf{X}\right)^{n_{1}} \cdots(\boldsymbol{\Gamma})^{m} \\
& +\lambda_{n_{1}, n_{2}, \cdots ; m} D_{\theta}\left(\left(D_{\theta} \mathbf{X}\right)^{n_{1}} \cdots\right)(\boldsymbol{\Gamma})^{m} \\
& \left.+(-1)^{m} \lambda_{n_{1}, n_{2}, \cdots ; m}\left(D_{\theta} \mathbf{X}\right)^{n_{1}}\left(D_{\theta} \mathbf{X}\right)^{n_{2}} \cdots \times D_{\theta}(\boldsymbol{\Gamma})^{m}\right)
\end{aligned}
$$

where $\lambda_{n_{1}, n_{2}, \cdots ; m}$ are the gauge transformation parameters with the appropriate reality conditions and the following condition: $\lambda=0$ if $\sum_{a=\text { odd }} n_{a}+m=2 \mathbf{Z}$. The last term has $D_{\theta}$ acting on $\boldsymbol{\Gamma}$. In order to maintain the usual kinetic term for the auxiliary superfields $\boldsymbol{\Gamma}$, we need to redefine them as follows:

$$
\boldsymbol{\Gamma}^{\prime i}=\boldsymbol{\Gamma}^{i}+2 i \frac{\partial}{\partial \boldsymbol{\Gamma}^{i}}\left(\lambda_{n_{1}, n_{2}, \cdots ; m}(\mathbf{X})\left(D_{\theta} \mathbf{X}\right)^{n_{1}}\left(D_{\theta} \mathbf{X}\right)^{n_{2}} \cdots \times(\boldsymbol{\Gamma})^{m}\right) .
$$

This field redefinition induces the following terms which are linear in $\lambda$ :

$$
-2 i\left(\frac{\partial}{\partial \boldsymbol{\Gamma}_{i}} A\right)\left(\frac{\partial}{\partial \boldsymbol{\Gamma}_{i}} \lambda\right)
$$

However, this is a naive expression because we need to consider composite operators by the nonzero correlators between $\Gamma$ 's. Taking this effect into account, instead of (4.11), we will have

$$
i[\lambda, A]=i(\lambda * A-A * \lambda),
$$

where $*$ represents the fermionic $*$-product defined in [19]. Thus, the gauge transformation is given by

$$
A \rightarrow A+d \lambda+i[\lambda, A]+e(\lambda)
$$

where

$$
e(\lambda)=\lambda_{n_{1}, n_{2}, \cdots ; m} D_{\theta}\left(\left(D_{\theta} \mathbf{X}\right)^{n_{1}} \cdots\right)(\boldsymbol{\Gamma})^{m} .
$$

By the localization, only the fields with $n_{a}=0(a>1)$ remain. The remaining fields precisely form the Quillen's superconnection $\mathcal{A}$. We will represent it as follows:

$$
\mathcal{A}=\mathcal{A}_{\mu_{1}, \cdots, \mu_{n}}^{k_{1}, \cdots, k_{m}} d x^{\mu_{1}} \cdots d x^{\mu_{n}} \gamma^{k_{1}} \cdots \gamma^{k_{m}}
$$

where we have replaced $\boldsymbol{\Gamma}^{k}$ to $\gamma^{k}$ which is the gamma matrix and $D_{\theta} \mathbf{X}^{\mu}$ to $d x^{\mu}$. The gauge transformation parameter is also given by

$$
\lambda=\lambda_{\mu_{1}, \cdots, \mu_{n}}^{k_{1}, \cdots, k_{m}} d x^{\mu_{1}} \cdots d x^{\mu_{n}} \gamma^{k_{1}} \cdots \gamma^{k_{m}}
$$


Then, the gauge transformation for the remaining fields is given by

$$
\mathcal{A} \rightarrow \mathcal{A}+d \lambda+i[\lambda, \mathcal{A}]
$$

Here the commutator using the fermionic $*$-product is identified as the supercommutator. The field strength defined by

$$
\mathcal{F}=i\left(D_{\mathcal{A}}\right)^{2}=d \mathcal{A}-i \mathcal{A}^{2},
$$

where $D_{\mathcal{A}} \equiv d-i \mathcal{A}$, and $\mathcal{F}$ is transformed as $\mathcal{F} \rightarrow \mathcal{F}+i[\lambda, \mathcal{F}]$. We see that $\operatorname{Str}(f(\mathcal{F}))$ is gauge invariant where Str is the supertrace for the superconnection.

Now, from the result for the BPS D-brane, it is almost clear that the cylinder partition function, which is the RR coupling of the D-branes and should be gauge-invariant by definition, is given by

$$
Z=2^{-5} \mathcal{N} \int \sum_{p=o d d}(-2 i)^{\frac{9-p}{2}} C^{(p+1)} \wedge \operatorname{Str}\left(e^{2 \pi i \mathcal{F}}\right)
$$

where we have replaced $\psi_{0}^{\mu}$ by $(-i) d x^{\mu}$, and $\mathcal{N}$ is the overall normalization, which will be fixed to $\mathcal{N}=2^{5} T_{D 9}$. This (4.19) is written by the super field strength of the superconnection, and our final result for the general Ramond-Ramond coupling of the D9-antiD9 branes.

We can derive the RR coupling (4.19) explicitly, as follows. The boundary action which will survive after the localization can be written as

$$
I_{\mathrm{B}}=-\int \mathrm{d} \tau \mathrm{d} \theta\left[\frac{1}{4} \boldsymbol{\Gamma}^{I} D_{\theta} \boldsymbol{\Gamma}^{I}+i \sum_{m=0}^{2 n} \mathcal{A}^{I_{1} \cdots I_{m}}\left(\mathbf{X}, D_{\theta} \mathbf{X}\right) \boldsymbol{\Gamma}^{I_{1}} \cdots \boldsymbol{\Gamma}^{I_{m}}\right] .
$$

For the evaluation of the $\boldsymbol{\Gamma}$ integral, we can use the identity shown in [19]

$$
\int D \boldsymbol{\Gamma} e^{\int \mathrm{d} \tau \mathrm{d} \theta\left[\frac{1}{4} \boldsymbol{\Gamma}^{I} D_{\theta} \boldsymbol{\Gamma}^{I}+\mathbf{M}(\boldsymbol{\Gamma})\right]}=\operatorname{Str} \mathrm{P} e^{\int d \tau\left(M_{1}(\gamma)-\left(M_{0}(\gamma)\right)^{2}\right)},
$$

where $\mathbf{M}(\boldsymbol{\Gamma})=M_{0}(\boldsymbol{\Gamma})+\theta M_{1}(\boldsymbol{\Gamma})$ in which only the superfield $\mathbf{X}$ was decomposed to the component fields. Here the r.h.s. of (4.21) is represented by the corresponding gamma matrix and $\mathrm{P}$ represents the path-ordering. Using this with the zero mode reduction by the localization, we have $M(\gamma)=i \mathcal{A}\left(X_{0}+i \theta \psi_{0}, i \psi_{0}\right)=i \mathcal{A}\left(X_{0}, i \psi_{0}\right)-\theta \psi_{0}^{\mu} \frac{\partial}{\partial X_{0}^{\mu}} \mathcal{A}\left(X_{0}, i \psi_{0}\right)$ and

$$
\int D \boldsymbol{\Gamma} e^{-I_{\mathrm{B}}}=\operatorname{Str} e^{2 \pi i \mathcal{F}}
$$

Therefore the general RR coupling formula is given by (4.19).

The expression (4.19) is consistent with the charge quantization, because the integral of Chern character of the super connection, $\operatorname{Str} \exp \left(\frac{\mathcal{F}}{2 \pi}\right)$, will be quantized. With the help of the relation among D-brane tensions $T_{D(p-2)} / T_{D p}=8 \pi^{2}$, one can show that the D-brane charge is quantized with our general result (4.19). 
In the RR coupling formula (4.19) the terms appearing in the exponent may look different from the standard normalization. This is just due to the convention. If we replace $\psi_{0}^{\mu}$ by $\sqrt{2 i} d x^{\mu}$ (instead of the previous $(-i) d x^{\mu}$ ), the $\mathrm{RR}$ coupling is rewritten by a formula with a more familiar Chern-Simons couplings as

$$
S_{\mathrm{RR}}(=Z)=T_{D 9} \int C \wedge \operatorname{Str}\left(e^{4 \pi \tilde{\mathcal{F}}}\right),
$$

where

$$
\tilde{\mathcal{F}} \equiv \sum_{n}(-2 i)^{\frac{n-1}{2}} \mathcal{F}^{(n+1)} .
$$

Here $\mathcal{F}=\sum_{n} \mathcal{F}^{(n+1)}$ and $\mathcal{F}^{(n+1)}$ is an $(n+1)$-form. Note that this replacement of $d x^{\mu}$ does not affect the result except for a change of the overall constant, because (4.19) contains the volume form only. In this formula we can see that the overall normalization is correct.

This $\tilde{\mathcal{F}}$ can also be considered as the field strength of a supperconnection. Indeed, with

$$
\tilde{\mathcal{A}}=\sum_{n}(-2 i)^{\frac{n-1}{2}} \mathcal{A}_{n}, \quad \tilde{\lambda}=\sum_{n}(-2 i)^{\frac{n}{2}} \lambda_{n},
$$

where $\mathcal{A}=\sum_{n} \mathcal{A}_{n}, \quad \lambda=\sum_{n} \lambda_{n}$, we have $\tilde{\mathcal{F}}=i(d-i \tilde{\mathcal{A}})^{2}$ and the gauge transformation is given by $\tilde{\mathcal{A}} \rightarrow \tilde{\mathcal{A}}+d \tilde{\lambda}+i[\tilde{\lambda}, \tilde{\mathcal{A}}]$.

Let us examine the reality condition. First, from the reality condition of the boundary action, we find a Hermiticity condition

$$
\left(\mathcal{A}_{\mu_{1}, \cdots, \mu_{n}}^{k_{1}, \cdots, k_{m}}\right)^{\dagger}=(-1)^{n+1+\frac{n+m-1}{2}}\left(\mathcal{A}_{\mu_{1}, \cdots, \mu_{n}}^{k_{1}, \cdots, k_{m}}\right),
$$

where we have used $n+m=2 \mathbf{Z}+1$ and also the following manipulation

$$
(i \mathcal{A})^{\dagger}=(-1)^{n+1+\frac{(n+m-1)(n+m)}{2}} i\left(\mathcal{A}_{\mu_{1}, \cdots, \mu_{n}}^{k_{1}, \cdots, k_{m}}\right)^{\dagger} D_{\theta} \mathbf{X}^{\mu_{1}} \cdots D_{\theta} \mathbf{X}^{\mu_{n}} \boldsymbol{\Gamma}^{k_{1}} \cdots \boldsymbol{\Gamma}^{k_{m}} .
$$

Now we consider the p-form valued $2^{n} \times 2^{n}$ matrix

$$
\mathcal{A}=\mathcal{A}_{\mu_{1}, \cdots, \mu_{n}}^{k_{1}, \cdots, k_{m}}(X) d x^{\mu_{1}} \cdots d x^{\mu_{n}} \gamma^{k_{1}} \cdots \gamma^{k_{m}}=\left(\begin{array}{cc}
A^{+}(X) & -i \bar{T}(X) \\
-i T(X) & A^{-}(X)
\end{array}\right),
$$

where in the r.h.s. of the equation we decomposed it to $2^{n-1} \times 2^{n-1}$ matrices. With this decomposition, we find ${ }^{12}$

$$
\left(A^{ \pm}\right)^{\dagger}=A^{ \pm}, \quad T^{\dagger}=\bar{T}
$$

with the following definition of the conjugate which acts on the forms,

$$
\left(d x^{\mu_{1}} \cdots d x^{\mu_{n}}\right)^{\dagger}=\left(d x^{\mu_{n}} \cdots d x^{\mu_{1}}\right) .
$$

\footnotetext{
${ }^{12}$ Let us explain how we take the conjugate of (4.28). We have $\left(\gamma^{k_{1}} \cdots \gamma^{k_{m}}\right)^{\dagger}=\gamma^{k_{1}} \cdots \gamma^{k_{m}}(-1)^{m(m-1) / 2}$ and $\left(d x^{\mu_{1}} \cdots d x^{\mu_{n}}\right)^{\dagger}=\left(d x^{\mu_{n}} \cdots d x^{\mu_{1}}\right)=\left(d x^{\mu_{1}} \cdots d x^{\mu_{n}}\right)(-1)^{n(n-1) / 2}$. So, in total, together with (4.26), the conjugate of the l.h.s. of (4.28) provides a factor $(-1)^{f}$ with $f=n+1+(n+m-1) / 2+m(m-1) / 2+n(n-1) / 2$. Noticing that $f=(n+1)+(n-1)(n+1) / 2+m^{2} / 2=1+n+n^{2} / 2+(m-1)(m+1) / 2$, we find $f$ is even (or odd) when $(m, n)=\left(\right.$ even, odd) (or (odd, even)). Therefore, the diagonal component $A^{ \pm}$(where odd forms appear) in the r.h.s. of (4.28) should be Hermitian, while $-i T$ and $-i \bar{T}$ (which are even forms) are related as $(-i T)^{\dagger}=+i \bar{T}$.
} 
Let us write a more explicit form of the RR coupling formula. As explained in [4], choosing an off-diagonal basis for gamma matrices, the matrix multiplication rule is given by

$$
\left(\begin{array}{ll}
A & B \\
C & D
\end{array}\right)\left(\begin{array}{ll}
A^{\prime} & B^{\prime} \\
C^{\prime} & D^{\prime}
\end{array}\right)=\left(\begin{array}{ll}
A A^{\prime}+(-)^{C^{\prime}} B C^{\prime} & A B^{\prime}+(-)^{D^{\prime}} B D^{\prime} \\
(-)^{A^{\prime}} C A^{\prime}+D C^{\prime} & (-)^{B^{\prime}} C B^{\prime}+D D^{\prime}
\end{array}\right),
$$

where $(-)^{A}$ is +1 or -1 if $A$ is bosonic or fermionic, respectively. (Here $d x^{\mu}$ is treated as a fermion, but $\gamma^{k}$ is treated as a boson.) Thus we have

$$
\begin{aligned}
2 \pi i \mathcal{F} & =2 \pi i\left(\mathrm{~d} \mathcal{A}-i \mathcal{A}^{2}\right) \\
& =2 \pi\left(\begin{array}{cc}
-\bar{T} T+i\left(\mathrm{~d} A^{+}-i A^{+} A^{+}\right) & \mathrm{d} \bar{T}-i A^{+} \bar{T}+i \bar{T} A^{-} \\
\mathrm{d} T-i A^{-} T+i T A^{+} & -T \bar{T}+i\left(\mathrm{~d} A^{-}-i A^{-} A^{-}\right)
\end{array}\right) .
\end{aligned}
$$

The RR coupling formula (4.19) is written with this curvature $\mathcal{F}$ of the superconnection, and here we provide an explicit form of $i \mathcal{F}$ as

$$
\begin{aligned}
-\bar{T} T & +i\left(\mathrm{~d} A^{+}-i A^{+} A^{+}\right) \\
= & -\bar{T}^{(0)} T^{(0)} \\
& +i\left(\mathrm{~d} A_{+}^{(1)}-i A_{+}^{(1)} \wedge A_{+}^{(1)}\right)-\bar{T}^{(0)} \wedge T^{(2)}-\bar{T}^{(2)} \wedge T^{(0)} \\
& +i\left(\mathrm{~d} A_{+}^{(3)}-i A_{+}^{(1)} \wedge A_{+}^{(3)}-i A_{+}^{(3)} \wedge A_{+}^{(1)}\right)-\bar{T}^{(0)} \wedge T^{(4)}-\bar{T}^{(4)} \wedge T^{(0)}-\bar{T}^{(2)} \wedge T^{(2)} \\
& +\cdots \\
\mathrm{d} T- & i A^{-} T+i T A^{+} \\
= & \mathrm{d} T^{(0)}-i A_{-}^{(1)} \wedge T^{(0)}+i T^{(0)} \wedge A_{+}^{(1)} \\
& +\mathrm{d} T^{(2)}-i A_{-}^{(3)} \wedge T^{(0)}+i T^{(0)} \wedge A_{+}^{(3)}-i A_{-}^{(1)} \wedge T^{(2)}+i T^{(2)} \wedge A_{+}^{(1)} \\
& +\cdots
\end{aligned}
$$

where $T^{(n)}$ represents the $n$-form part. Substitution of these expression explicitly with $\operatorname{Str}(*)=\operatorname{Tr}\left(\sigma_{3}(*)\right)$ provides the RR coupling.

Instead of $\mathcal{A}$, we can use $\tilde{\mathcal{A}}$, which may be more physical. For this, it would be more convenient to use following another definition of the conjugate acting trivially on the forms: $\left(d x^{\mu_{1}} \cdots d x^{\mu_{n}}\right)^{\dagger}=\left(d x^{\mu_{1}} \cdots d x^{\mu_{n}}\right)$. With this and the non-trivial factor appeared in the transformation from $\mathcal{A}$ to $\tilde{\mathcal{A}}$, we see that

$$
\left(\tilde{A}^{ \pm}\right)^{\dagger}=\tilde{A}^{ \pm}, \quad \tilde{T}^{\dagger}=\overline{\tilde{T}}
$$

for

$$
\tilde{\mathcal{A}}=\tilde{\mathcal{A}}_{\mu_{1}, \cdots, \mu_{n}}^{k_{1}, \cdots, \mu_{m}}(X) d x^{\mu_{1}} \cdots d x^{\mu_{n}} \gamma^{k_{1}} \cdots \gamma^{k_{m}}=\left(\begin{array}{cc}
\tilde{A}^{+}(X) & i^{\frac{3}{2}} \overline{\tilde{T}}(X) \\
i^{\frac{3}{2}} \tilde{T}(X) & \tilde{A}^{-}(X)
\end{array}\right) .
$$

The generalization to the $\mathrm{D} p$-antiD $p$ brane system can be done following [5, 19] with the T-dualized formula. In this system the RR-charges are known to be generated by the ascent relation $[57,58]$ by the tachyon condensation and there would be analogue of this for the massive fields. Our RR-charge formula is written using the supercommutator which 
can be generalized to arbitrary numbers of $\mathrm{D} p$-branes and anti $\mathrm{D} p$-branes, in particular, which include the system with the BPS Dp-branes only. The validity of this procedure can be shown by taking the some D-branes infinitely far away or considering the infinitely many D-anti-D-brane system realized by the Gamma matrix of $\mathrm{SO}(\infty)$ with the tachyon condensation which gives any number of D-branes. Note that, of course, restricting the super connection $\mathcal{A}$ to its upper-left corner, we trivially reproduce the (the non-Abelian generalization of) RR coupling formula (4.4) of the BPS D-brane.

\section{Summary and discussion}

In this paper, we found the most general RR coupling formula of D-branes. We allowed arbitrary number of insertions of all massive excitations of open superstring theory. The worldsheet theory is the 2-dimensional $\mathcal{N}=(1,1)$ supersymmetric field theory with free chiral multiplets. The worldsheet was taken to be a cylinder, and at one boundary we considered arbitrary boundary deformations preserving a half of the supersymmetries, and the other boundary corresponds to the Ramond-Ramond bulk vertex. The localization technique is powerful enough to evaluate the cylinder partition function with these boundary conditions, and it is nothing but the RR coupling formula in view of boundary superstring field theory (BSFT).

We considered BPS D9-branes and also the case of pairs of a D9 and an antiD9. In either case, the RR charge formula is written in a simple manner with Quillen's superconnection, (4.19). Interestingly, only a finite number of massive excitation fields can enter the formula, and the resultant Chern characters of the superconnection can make sure the RR charge quantization.

We have used a cylinder worldsheet, instead of a disk worldsheet which has been commonly used in BSFT. Instead, we used the infinitely long cylinder which is an alternative flat worldsheet, to formulate the off-shell supersymmetries consistent with the standard worldsheet theory. In any case, the shape of the worldsheet does not give physical difference for the result, since normally the deformation of the shape should correspond to a field redefinition on the worldsheet boundary fields.

In this paper we concentrated on the RR coupling formula. From the viewpoint of the BSFT, equally important action is for the Neveu-Schwarz Neveu-Schwarz (NSNS) sector which describes couplings of all the off-shell open superstring modes to the bulk gravity. To describe the NSNS sector, one needs a different quantization of fermion fields on the worldsheet.

We showed also that it is impossible to create of a D9-brane by a condensation of massive modes on a BPS D9-brane or on a D9-antiD9 pair. Our observation is in a good contrast with a recent report [59] on multiple D-brane solutions in cubic open string field theory [60]. Since no good relation (field redefinition) between the BSFT and the cubic open string field theory, it is plausible that the theory configuration space allowed for each theory is different from the first place. ${ }^{13}$ It would be interesting to find out where the

\footnotetext{
${ }^{13}$ We can think a BPS D-brane as a configuration in the infinitely many D9-antiD9-brane pairs with the nonzero tachyon. Then, in this point of view, a creation of the any number of D9-branes can be possible in the BSFT.
} 
discrepancy comes from, and a more precise understanding of the off-shell configuration space of superstring theory.

Our most general Ramond-Ramond coupling formula is written by higher form fields. It is interesting that the higher Chern characters of massless gauge field strengths are combined with the higher form fields on an equal footing. Our work showed for the first time that the open string higher forms, which are massive excitations, are relevant to extended objects in string theory - D-branes. In view of recent progress on generic study of higher form fields [61], it is important to study the higher structure of open string theory further.

\section{Acknowledgments}

S. S. would like to thank Satoshi Yamaguchi for discussions. S. T. would like to thank Shigeki Sugimoto for a useful comment. The work of K. H. is partially supported by the RIKEN iTHES project. The work of S. S. is supported by the JSPS Fellowship for Young Scientists. The work of S. T. is partly supported by JSPS KAKENHI Grant Number 23740189 .

Open Access. This article is distributed under the terms of the Creative Commons Attribution License (CC-BY 4.0), which permits any use, distribution and reproduction in any medium, provided the original author(s) and source are credited.

\section{References}

[1] J. Polchinski, Dirichlet Branes and Ramond-Ramond charges, Phys. Rev. Lett. 75 (1995) 4724 [hep-th/9510017] [INSPIRE].

[2] M. Li, Boundary states of D-branes and Dy strings, Nucl. Phys. B 460 (1996) 351 [hep-th/9510161] [InSPIRE].

[3] M.R. Douglas, Branes within branes, in Strings, branes and dualities, Cargese France (1997), pg. 267 [hep-th/9512077] [INSPIRE].

[4] P. Kraus and F. Larsen, Boundary string field theory of the D $\bar{D}$ system, Phys. Rev. D 63 (2001) 106004 [hep-th/0012198] [INSPIRE].

[5] T. Takayanagi, S. Terashima and T. Uesugi, Brane-antibrane action from boundary string field theory, JHEP 03 (2001) 019 [hep-th/0012210] [INSPIRE].

[6] E. Witten, On background independent open-string field theory, Phys. Rev. D 46 (1992) 5467 [hep-th/9208027] [INSPIRE].

[7] E. Witten, Some computations in background independent off-shell string theory, Phys. Rev. D 47 (1993) 3405 [hep-th/9210065] [INSPIRE].

[8] S.L. Shatashvili, Comment on the background independent open string theory, Phys. Lett. B 311 (1993) 83 [hep-th/9303143] [INSPIRE].

[9] S.L. Shatashvili, On the problems with background independence in string theory, Alg. Anal. 6 (1994) 215 [hep-th/9311177] [INSPIRE]. 
[10] D. Kutasov, M. Mariño and G.W. Moore, Remarks on tachyon condensation in superstring field theory, hep-th/0010108 [INSPIRE].

[11] M. Mariño, On the BV formulation of boundary superstring field theory, JHEP 06 (2001) 059 [hep-th/0103089] [INSPIRE].

[12] V. Niarchos and N. Prezas, Boundary superstring field theory, Nucl. Phys. B 619 (2001) 51 [hep-th/0103102] [INSPIRE].

[13] O.D. Andreev and A.A. Tseytlin, Partition function representation for the open superstring effective action: cancellation of Mobius infinities and derivative corrections to Born-Infeld Lagrangian, Nucl. Phys. B 311 (1988) 205 [INSPIRE].

[14] A. Sen, Tachyon condensation on the brane antibrane system, JHEP 08 (1998) 012 [hep-th/9805170] [INSPIRE].

[15] A. Sen, Descent relations among bosonic D-branes, Int. J. Mod. Phys. A 14 (1999) 4061 [hep-th/9902105] [INSPIRE].

[16] A. Sen, Non-BPS states and Branes in string theory, hep-th/9904207 [INSPIRE].

[17] A. Sen, Universality of the tachyon potential, JHEP 12 (1999) 027 [hep-th/9911116] [INSPIRE].

[18] A. Sen, Tachyon dynamics in open string theory, Int. J. Mod. Phys. A 20 (2005) 5513 [hep-th/0410103] [INSPIRE].

[19] T. Asakawa, S. Sugimoto and S. Terashima, Exact description of D-branes via tachyon condensation, JHEP 02 (2003) 011 [hep-th/0212188] [INSPIRE].

[20] K. Hashimoto and S. Terashima, Boundary string field theory as a field theory: mass spectrum and interaction, JHEP 10 (2004) 040 [hep-th/0408094] [INSPIRE].

[21] K. Hashimoto and M. Murata, A landscape in boundary string field theory: new class of solutions with massive state condensation, PTEP 2013 (2013) 043B01 [arXiv:1211.5949] [INSPIRE].

[22] V. Pestun, Localization of gauge theory on a four-sphere and supersymmetric Wilson loops, Commun. Math. Phys. 313 (2012) 71 [arXiv:0712.2824] [inSPIRE].

[23] E. Witten, Topological Quantum Field Theory, Commun. Math. Phys. 117 (1988) 353 [INSPIRE].

[24] N.A. Nekrasov, Seiberg-Witten prepotential from instanton counting, Adv. Theor. Math. Phys. 7 (2004) 831 [hep-th/0206161] [INSPIRE].

[25] A. Kapustin, B. Willett and I. Yaakov, Exact results for Wilson loops in superconformal Chern-Simons theories with matter, JHEP 03 (2010) 089 [arXiv:0909.4559] [INSPIRE].

[26] D.L. Jafferis, The exact superconformal R-symmetry extremizes Z, JHEP 05 (2012) 159 [arXiv: 1012.3210] [INSPIRE].

[27] N. Hama, K. Hosomichi and S. Lee, Notes on SUSY gauge theories on three-sphere, JHEP 03 (2011) 127 [arXiv: 1012.3512] [INSPIRE].

[28] N. Hama, K. Hosomichi and S. Lee, SUSY gauge theories on squashed three-spheres, JHEP 05 (2011) 014 [arXiv: 1102.4716] [inSPIRE].

[29] G. Festuccia and N. Seiberg, Rigid supersymmetric theories in curved superspace, JHEP 06 (2011) 114 [arXiv:1105.0689] [INSPIRE]. 
[30] Y. Imamura, Relation between the $4 d$ superconformal index and the $S^{3}$ partition function, JHEP 09 (2011) 133 [arXiv: 1104.4482] [INSPIRE].

[31] J. Källén and M. Zabzine, Twisted supersymmetric 5D Yang-Mills theory and contact geometry, JHEP 05 (2012) 125 [arXiv:1202.1956] [INSPIRE].

[32] K. Hosomichi, R.-K. Seong and S. Terashima, Supersymmetric gauge theories on the five-sphere, Nucl. Phys. B 865 (2012) 376 [arXiv:1203.0371] [INSPIRE].

[33] F. Benini and S. Cremonesi, Partition functions of $\mathcal{N}=(2,2)$ gauge theories on $S^{2}$ and vortices, Commun. Math. Phys. 334 (2015) 1483 [arXiv:1206.2356] [InSPIRE].

[34] N. Doroud, J. Gomis, B. Le Floch and S. Lee, Exact results in $D=2$ supersymmetric gauge theories, JHEP 05 (2013) 093 [arXiv:1206.2606] [INSPIRE].

[35] T. Kawano and N. Matsumiya, $5 D S Y M$ on $3 D$ sphere and $2 D Y M$, Phys. Lett. B 716 (2012) 450 [arXiv: 1206.5966] [INSPIRE].

[36] J. Källén, J. Qiu and M. Zabzine, The perturbative partition function of supersymmetric $5 D$ Yang-Mills theory with matter on the five-sphere, JHEP 08 (2012) 157 [arXiv:1206.6008] [INSPIRE].

[37] H.-C. Kim and S. Kim, M5-branes from gauge theories on the 5-sphere, JHEP 05 (2013) 144 [arXiv: 1206.6339] [INSPIRE].

[38] N. Hama and K. Hosomichi, Seiberg-Witten theories on ellipsoids, JHEP 09 (2012) 033 [Addendum ibid. 10 (2012) 051] [arXiv: 1206 .6359] [inSPIRE].

[39] S. Terashima, Supersymmetric gauge theories on $S^{4} \times S^{1}$, Phys. Rev. D 89 (2014) 125001 [arXiv: 1207.2163] [INSPIRE].

[40] Y. Asano, G. Ishiki, T. Okada and S. Shimasaki, Exact results for perturbative partition functions of theories with $\mathrm{SU}(2 \mid 4)$ symmetry, JHEP 02 (2013) 148 [arXiv:1211.0364] [INSPIRE].

[41] H. Kim, S. Lee and P. Yi, Exact partition functions on $\mathbb{R P}^{2}$ and orientifolds, JHEP 02 (2014) 103 [arXiv:1310.4505] [INSPIRE].

[42] T. Nosaka and S. Terashima, Supersymmetric gauge theories on a squashed four-sphere, JHEP 12 (2013) 001 [arXiv:1310.5939] [INSPIRE].

[43] S. Terashima, A localization computation in confining phase, arXiv:1410.3630 [INSPIRE].

[44] S. Sugishita and S. Terashima, Exact results in supersymmetric field theories on manifolds with boundaries, JHEP 11 (2013) 021 [arXiv:1308.1973] [INSPIRE].

[45] D. Honda and T. Okuda, Exact results for boundaries and domain walls in $2 d$ supersymmetric theories, arXiv:1308.2217 [INSPIRE].

[46] K. Hori and M. Romo, Exact results in two-dimensional (2,2) supersymmetric gauge theories with boundary, arXiv:1308.2438 [INSPIRE].

[47] Y. Yoshida and K. Sugiyama, Localization of $3 d \mathcal{N}=2$ supersymmetric theories on $S^{1} \times D^{2}$, arXiv:1409.6713 [INSPIRE].

[48] M.B. Green, J.A. Harvey and G.W. Moore, I-brane inflow and anomalous couplings on D-branes, Class. Quant. Grav. 14 (1997) 47 [hep-th/9605033] [INSPIRE].

[49] J. Halverson, H. Jockers, J.M. Lapan and D.R. Morrison, Perturbative corrections to Kähler moduli spaces, Commun. Math. Phys. 333 (2015) 1563 [arXiv:1308.2157] [InSPIRE]. 
[50] D. Quillen, Superconnections and the Chern character, Topology 24 (1985) 89 [INSPIRE].

[51] N. Berline, E. Getzler and M. Vergne, Heat kernels and Dirac operators, Springer-Verlag (1991).

[52] C. Kennedy and A. Wilkins, Ramond-Ramond couplings on brane-antibrane systems, Phys. Lett. B 464 (1999) 206 [hep-th/9905195] [INSPIRE].

[53] D. Kutasov, M. Mariño and G.W. Moore, Remarks on tachyon condensation in superstring field theory, hep-th/0010108 [INSPIRE].

[54] J.A. Harvey, D. Kutasov and E.J. Martinec, On the relevance of tachyons, hep-th/0003101 [INSPIRE].

[55] A.A. Tseytlin, $\sigma$-model approach to string theory effective actions with tachyons, J. Math. Phys. 42 (2001) 2854 [hep-th/0011033] [INSPIRE].

[56] E. Witten, D-branes and k-theory, JHEP 12 (1998) 019 [hep-th/9810188] [INSPIRE].

[57] T. Asakawa, S. Sugimoto and S. Terashima, D-branes, matrix theory and K homology, JHEP 03 (2002) 034 [hep-th/0108085] [INSPIRE].

[58] S. Terashima, A Construction of commutative D-branes from lower dimensional nonBPS D-branes, JHEP 05 (2001) 059 [hep-th/0101087] [INSPIRE].

[59] T. Erler and C. Maccaferri, String field theory solution for any open string background, JHEP 10 (2014) 029 [arXiv: 1406.3021] [INSPIRE].

[60] E. Witten, Noncommutative Geometry and String Field Theory, Nucl. Phys. B 268 (1986) 253 [INSPIRE].

[61] D. Gaiotto, A. Kapustin, N. Seiberg and B. Willett, Generalized global symmetries, JHEP 02 (2015) 172 [arXiv: 1412.5148] [INSPIRE]. 